\title{
On the decay of dispersive motions in the outer region of rough-wall boundary layers
}

\author{
Johan Meyers $^{1} \dagger$, Bharathram Ganapathisubramani ${ }^{2}$ and \\ Raúl Bayoán $\mathrm{Cal}^{3}$ \\ ${ }^{1}$ KU Leuven, Mechanical Engineering, Celestijnenlaan 300, B3001 Leuven, Belgium \\ ${ }^{2}$ University of Southampton, Aerodynamics \& Flight Mechanics Group, \\ Southampton SO17 1BJ, United Kingdom \\ ${ }^{3}$ Portland State University, Mechanical and Materials Engineering, Portland, OR 97207, \\ United States
}

(Received xx; revised xx; accepted xx)

In rough-wall boundary layers, wall-parallel non-homogeneous mean-flow solutions exist that lead to so-called dispersive velocity components and dispersive stresses. They play a significant role in the mean-flow momentum balance near the wall, but typically disappear in the outer layer. A theoretical framework is presented to study the decay of dispersive motions in the outer layer. To this end, the problem is formulated in Fourier space, and a set of governing ordinary differential equations per mode in wavenumber space is derived by linearizing the Reynolds-averaged Navier-Stokes equations around a constant background velocity. With further simplifications, analytically tractable solutions are found consisting of linear combinations of $\exp (-k z)$ and $\exp (-K z)$, with $z$ the wall distance, $k$ the magnitude of the horizontal wavevector $\boldsymbol{k}$, and where $K(\boldsymbol{k}, R e)$ is a function of $\boldsymbol{k}$ and the Reynolds number Re. Moreover, for $k \rightarrow \infty$ or $k_{1} \rightarrow 0$ (with $k_{1}$ the stream-wise wavenumber), $K \rightarrow k$ is found, in which case solutions consist of a linear combination of $\exp (-k z)$ and $z \exp (-k z)$, and are Reynolds number independent. These analytical relations are compared in the limit of $k_{1}=0$ to the rough boundary layer experiments by Vanderwel and Ganapathisubramani (J. Fluid Mech. 774, R2, 2015) and are in reasonable agreement for $\ell_{k} / \delta \leqslant 0.5$, with $\delta$ the boundary-layer thickness and $\ell_{k}=2 \pi / k$.

\section{Introduction}

When analyzing turbulent flow over rough surfaces, flow statistics are often averaged over wall-parallel planes. The difference between mean flow and horizontally averaged mean flow yields dispersive velocities, and leads to so-called dispersive or coherent stresses in the horizontally averaged mean momentum equations (Raupach \& Shaw 1982; Finnigan 2000; Nikora et al. 2007). The effects of the shape and distribution of surface roughness on turbulent wall-flows is typically assumed to be confined to the roughness sub-layer where the dispersive motions are dominant and complement Reynolds shear stresses (Raupach et al. 1991; Manes et al. 2008). In this region, the mean flow statistics are complex and three dimensional, governed by the complex shape of the roughness itself.

In flows over homogeneous rough surfaces, the roughness sub-layer is typically found to extend vertically 2-5 times the representative roughness heights, which can be the equivalent sandgrain roughness or the maximum roughness height or a root-mean-square

$\dagger$ Email address for correspondence: johan.meyers@kuleuven.be 
roughness height, depending on the type of roughness (Raupach et al. 1991; Nikora et al. 2001; Jiménez 2004; Flack et al. 2007). When moving away from the wall, the dispersive stress contribution to the total stress gradually decays, in accordance with the picture of an outer layer that is dominated by Reynolds stresses. Therefore, beyond this roughness sub-layer, the outer layer of the flow is usually independent of local details of surface roughness, resulting in a mean flow that is nearly homogeneous in wall-parallel directions, with flow statistics that mainly depend on the wall-normal direction (Townsend 1956, 1976; Castro 2007). However, for rough surfaces with spatial heterogeneities where dominant spanwise length scales of the roughness distribution are on the order of the outer length scale of the flow, large secondary motions are excited by the roughness arrangement, and can penetrate into the outer layer (Nezu \& Nakagawa 1984; Wang \& Cheng 2005; Barros \& Christensen 2014; Anderson et al. 2015; Vanderwel \& Ganapathisubramani 2015; Kevin et al. 2017; Medjnoun et al. 2018; Hwang \& Lee 2018). Therefore, dispersive stress can be significant across the entire turbulent layer.

Recent work has shown that the decay of dispersive stresses (or secondary motions) scales with the spanwise roughness wavelength when the roughness is geometrically scaled (proportional increase in both roughness height and wavelength) and when the wavelength increases at fixed roughness height (Yang \& Anderson 2018; Chan et al. 2018). Very recently, Morgan \& McKeon (2018) analyzed the spatial and wave-number structure of dispersive motions in a boundary layer with periodic roughess elements. In the current work, a new analytical framework is proposed that allows to study this decay of dispersive motions systematically. The paper is organised as follows. First in Section 2, the theory is presented, and approximate solutions for dispersive motions in the outer layer of a boundary layer are derived. Next, a comparison with experiments is shown in Section 3. Lastly, discussion and conclusions are stated in Section 4.

\section{Approximate solutions for the dispersive velocity field}

\subsection{Horizontally-averaged Navier-Stokes equations and linearization}

Consider an incompressible turbulent boundary layer over a rough wall, with $x_{1}$ oriented in streamwise, $x_{2}$ in spanwise, and $x_{3}$ in wall-normal direction. Further, $\overline{\boldsymbol{u}}$ represents the Reynolds-averaged velocity field, with fluctuation $\boldsymbol{u}^{\prime}$. The focus is on rough boundary layers with either periodic roughness elements or a roughness distribution that is statistically homogeneous in horizontal directions, and the horizontally averaged and Reynolds-averaged flow is denoted with $\langle\overline{\boldsymbol{u}}\rangle \triangleq(U, 0, W)$. Furthermore, $\overline{\boldsymbol{u}}^{\prime \prime}$ is introduced, so that $\overline{\boldsymbol{u}}=U \boldsymbol{e}_{1}+W \boldsymbol{e}_{3}+\overline{\boldsymbol{u}}^{\prime \prime}$.

It is further presumed that the boundary layer is sufficiently developed for the streamwise evolution of mean velocity components to be negligible, so that the time-averaged and horizontally averaged Navier-Stokes equation follows as

$$
W \frac{\partial U}{\partial x_{3}}-\nu \frac{\partial^{2} U}{\partial x_{3}^{2}}+\frac{1}{\rho} \frac{\partial p_{\infty}}{\partial x_{1}}=-\frac{\partial\left\langle\overline{u_{1}^{\prime} u_{3}^{\prime}}\right\rangle}{\partial x_{3}}-\frac{\partial\left\langle\bar{u}_{1}^{\prime \prime} \bar{u}_{3}^{\prime \prime}\right\rangle}{\partial x_{3}}
$$

with $\left\langle\overline{u_{1}^{\prime} u_{3}^{\prime}}\right\rangle$ and $\left\langle\bar{u}_{1}^{\prime \prime} \bar{u}_{3}^{\prime \prime}\right\rangle$ being the plane averaged Reynolds stress and dispersive stress respectively, $p_{\infty}$ the background pressure, $\nu$ the kinematic viscosity, and where the density $\rho$ is presumed to be constant.

The equations for the dispersive velocity fluctuations $\overline{\boldsymbol{u}}^{\prime \prime}$ further follow from sub- 
tracting (2.1) from the standard Navier-Stokes equations, yielding

$$
\begin{aligned}
& \frac{\partial \bar{u}_{i}^{\prime \prime}}{\partial x_{i}}=0 \\
& U \frac{\partial \bar{u}_{i}^{\prime \prime}}{\partial x_{1}}+\bar{u}_{j}^{\prime \prime} \frac{\partial \bar{u}_{i}^{\prime \prime}}{\partial x_{j}}+\bar{u}_{3}^{\prime \prime} \Gamma \delta_{i 1}=-\frac{1}{\rho} \frac{\partial \bar{p}^{\prime \prime}}{\partial x_{i}}+\frac{\partial\left\langle\overline{u_{1}^{\prime} u_{3}^{\prime}}\right\rangle}{\partial x_{3}} \delta_{i 1}+\frac{\partial\left\langle\bar{u}_{1}^{\prime \prime} \bar{u}_{3}^{\prime \prime}\right\rangle}{\partial x_{3}} \delta_{i 1}-\frac{\partial \overline{u_{i}^{\prime} u_{j}^{\prime}}}{\partial x_{j}}+\nu \frac{\partial^{2} u_{i}^{\prime \prime}}{\partial x_{j} x_{j}} .
\end{aligned}
$$

Here, terms with products of $W$ and $\bar{u}_{i}^{\prime \prime}$ are considered as being negligible, and the short-hand notation $\Gamma=\partial U / \partial x_{3}$ is used.

For $\overline{\boldsymbol{u}}^{\prime \prime}$ sufficiently small, the dispersive velocity equations can be linearized around the mean background flow, neglecting all higher order terms. This leads to

$$
\begin{array}{r}
\frac{\partial \bar{u}_{i}^{\prime \prime}}{\partial x_{i}}=0, \\
U \frac{\partial \bar{u}_{i}^{\prime \prime}}{\partial x_{1}}+\bar{u}_{3}^{\prime \prime} \Gamma \delta_{i 1}=-\frac{1}{\rho} \frac{\partial \bar{p}^{\prime \prime}}{\partial x_{i}}-\frac{\partial\left(\overline{u_{i}^{\prime} u_{j}^{\prime}}\right)^{\prime \prime}}{\partial x_{j}}+\nu \frac{\partial^{2} u_{i}^{\prime \prime}}{\partial x_{j} x_{j}} .
\end{array}
$$

In particular, the solution of these equations are investigated in the outer layer, since we expect $\overline{\boldsymbol{u}}^{\prime \prime}$ to be small for $x_{3} \rightarrow \delta$, with $\delta$ the boundary layer thickness.

\subsection{Turbulence closure}

In order to solve (2.5), a closure is required for the Reynolds stresses $R_{i j}^{\prime \prime}=\left(\overline{u_{i}^{\prime} u_{j}^{\prime}}\right)^{\prime \prime}$. To this end, first, a simple closure for the background flow is posed. Providing that conditions for the linearization hold, it is reasonable to assume that the background corresponds to a standard outer layer solution in the absence of any dispersive terms. Pertaining to the Reynolds forces, an exact parametrization then corresponds to

$$
F_{i} \triangleq-\frac{\partial}{\partial x_{j}}\left(R_{i j}-\frac{1}{3} \delta_{i j} R_{k k}\right)=\frac{\partial}{\partial x_{j}} \nu_{e}\left(\frac{\partial \bar{u}_{i}}{\partial x_{j}}+\frac{\partial \bar{u}_{i}}{\partial x_{j}}\right)=\delta_{i 1} \frac{\partial \nu_{e} \Gamma}{\partial x_{3}},
$$

where as usual, the trace of the Reynolds stress is absorbed in the pressure term, and the eddy viscosity $\nu_{e}$ is straightforwardly determined from $\nu_{e}=-\left\langle\overline{u_{1}^{\prime} u_{3}^{\prime}}\right\rangle / \Gamma$, using known experimental, numerical or analytical profiles for $\Gamma$ and $\overline{u_{1}^{\prime} u_{3}^{\prime}}$ in the outer layer of a boundary layer. For later use in $\S 2.3$, the total viscous force is introduced as $G_{i} \triangleq$ $F_{i}+\partial / \partial x_{j}\left[\nu\left(\partial \bar{u}_{i} / \partial x_{j}+\partial \bar{u}_{i} / \partial x_{j}\right)\right]$.

The linearization of the Reynolds forces now follows from the chain rule as

$$
F_{i}^{\prime \prime}=\frac{\partial}{\partial x_{j}}\left[\nu_{e}\left(\frac{\partial \bar{u}_{i}^{\prime \prime}}{\partial x_{j}}+\frac{\partial \bar{u}_{i}^{\prime \prime}}{\partial x_{j}}\right)+\delta_{i 1} \delta_{j 3} \nu_{e}^{\prime \prime} \Gamma\right] .
$$

Unfortunately, the dispersive turbulent viscosity $\nu_{e}^{\prime \prime}$ is not known. However, when $\Gamma \ll 1$, which is generally true in boundary layers for $x_{3} \rightarrow \delta$, the term with $\nu_{e}^{\prime \prime} \Gamma$ disappears. Alternatively, this result is also obtained by linearizing the Navier-Stokes equations around a constant background velocity $U_{\infty}$ instead of $U$. The resulting equations are the same as (2.5), but with $U_{\infty}$ instead of $U$ and $\Gamma=0$. The linearization in that case is valid, as long as $\left|U_{\infty}-U\right| \ll 1$, which again holds for $x_{3} \rightarrow \delta$. Thus, for this particular case, (2.7) with $\Gamma=0$ yields an exact closure of the linearized Reynolds forces. Finally, it should be noted that the above proposed closure is not exact in terms of the Reynolds stresses themselves. This closure determines the Reynolds stresses up to an addition of a divergence-free tensor (see, e.g., Deser 1967; Wu et al. 1996; Jiménez 2016). The latter will have no influence on the Reynolds force, but will change the individual stress 
components. In particular, it is well understood that a classical eddy viscosity model leads to a stress tensor with a zero diagonal (corresponding to $\overline{u_{1}^{\prime} u_{1}^{\prime}}=\overline{u_{2}^{\prime} u_{2}^{\prime}}=\overline{u_{3}^{\prime} u_{3}^{\prime}}$ ), which is generally not the case in boundary layers. Here, this is not an issue, as the Reynolds stresses are not directly needed in the remainder of this work.

\subsection{Representation using Fourier modes}

Given periodic roughness elements, the linearized Navier-Stokes equations can be solved using periodic boundaries in $x_{1}$ and $x_{2}$ directions. Thus, solutions can be expressed based on a Fourier series. To this end,

$$
\begin{aligned}
\bar{u}_{i}^{\prime \prime} & =\sum_{\boldsymbol{k}} \widetilde{u}_{i}(\boldsymbol{k}, z) \exp \left(\imath\left(k_{1} x_{1}+k_{2} x_{2}\right)\right), \\
\bar{p}^{\prime \prime} & =\sum_{\boldsymbol{k}} \widetilde{p}(\boldsymbol{k}, z) \exp \left(\imath\left(k_{1} x_{1}+k_{2} x_{2}\right)\right),
\end{aligned}
$$

etc., are introduced with $\boldsymbol{k}=\left(k_{1}, k_{2}\right)$, and $z \triangleq x_{3}$. Furthermore, $k_{1}=i 2 \pi / L_{x}, k_{2}=$ $j 2 \pi / L_{y}$, with $i, j \in \mathbb{Z}$. We note that in the case of a roughness distribution, which is statistically homogeneous in horizontal directions (instead of periodic roughness elements), the above Fourier series can be replaced by Fourier integrals in horizontal planes, without further affecting results below. In this case, it is assumed that the largest horizontal length scales in the roughness distributions are sufficiently small for the streamwise homogeneity assumption of the dispersive-flow equations $(2.2,2.3)$ to hold.

Since solving linear equations is the aim, solutions can now be found mode by mode. To this end, the continuity equation is first eliminated by using $\widetilde{u}_{3}$ and $\widetilde{\omega}_{3}=-\imath k_{2} \widetilde{u}_{1}+\imath k_{1} \widetilde{u}_{2}$ as independent variables. Thus for $\left(k_{1}, k_{2}\right) \neq(0,0)$,

$$
\begin{aligned}
& \widetilde{u}_{1}=\frac{\imath k_{1}}{k^{2}} \frac{\mathrm{d} \widetilde{u}_{3}}{\mathrm{~d} z}+\frac{\imath k_{2}}{k^{2}} \widetilde{\omega}_{3}, \\
& \widetilde{u}_{2}=\frac{\imath k_{2}}{k^{2}} \frac{\mathrm{d} \widetilde{u}_{3}}{\mathrm{~d} z}-\frac{\imath k_{1}}{k^{2}} \widetilde{\omega}_{3},
\end{aligned}
$$

with $k=\left(k_{1}^{2}+k_{2}^{2}\right)^{1 / 2}$.

Inserting (2.10) and (2.11) in the linearized momentum equations (2.5), and further eliminating the pressure, then leads to following set of equations

$$
\begin{aligned}
&-k_{1} U \widetilde{\omega}_{3}+k_{2} \widetilde{u}_{3} \Gamma=k_{2} \tilde{G}_{1}-k_{1} \tilde{G}_{2}, \\
& k_{1} U \frac{\mathrm{d}^{2} \widetilde{u}_{3}}{\mathrm{~d} z^{2}}-k_{1} \widetilde{u}_{3} \frac{\mathrm{d} \Gamma}{\mathrm{d} z}-k_{1} U k^{2} \widetilde{u}_{3}=-k_{1} \frac{\mathrm{d} \tilde{G}_{1}}{\mathrm{~d} z}-k_{2} \frac{\mathrm{d} \tilde{G}_{2}}{\mathrm{~d} z}+\imath k^{2} \tilde{G}_{3},
\end{aligned}
$$

which constitutes a set of two coupled ordinary differential equations. Finally, using (2.7) and $\Gamma=0$ into above equations, using $U \approx U_{\infty}$, and some straightforward but cumbersome algebraic manipulations, leads to

$$
\begin{aligned}
& \frac{\mathrm{d}}{\mathrm{d} z} \nu_{t} \frac{\mathrm{d} \widetilde{\omega}_{3}}{\mathrm{~d} z}-\left(\imath U_{\infty} k_{1}+\nu_{t} k^{2}\right) \widetilde{\omega}_{3}=0 \\
& \frac{\mathrm{d}^{2}}{\mathrm{~d} z^{2}} \nu_{t} \frac{\mathrm{d}^{2} \widetilde{u}_{3}}{\mathrm{~d} z^{2}}-\frac{\mathrm{d}}{\mathrm{d} z}\left[\left(\imath k_{1} U_{\infty}+2 k^{2} \nu_{t}\right) \frac{\mathrm{d} \widetilde{u}_{3}}{\mathrm{~d} z}\right]+\left(\imath k_{1} U_{\infty}+k^{2} \nu_{t}+\frac{\mathrm{d}^{2} \nu_{t}}{\mathrm{~d} z^{2}}\right) k^{2} \widetilde{u}_{3}=0,
\end{aligned}
$$

with $\nu_{t}=\nu+\nu_{e}$ the total viscosity. For a classical developing boundary layer, boundary conditions at $z=\infty$ correspond to $\widetilde{\omega}_{3}(\infty)=0, \widetilde{u}_{3}(\infty)=0$, and $\mathrm{d} \widetilde{u}_{3} /\left.\mathrm{d} z\right|_{z=\infty}=0$. Three more boundary conditions are required to uniquely determine solutions. They should be given at a location $z$ which is sufficiently far from the wall for the linearized equations 
to hold. These additional conditions are not a priori known, and depend on the shape of the wall roughness, and the nonlinear dynamics of the flow close to the wall.

\subsection{Analytical solutions}

Given an outer layer parametrization of $\nu_{e}(z)$, and appropriate boundary conditions, $(2.14,2.15)$ can be solved. Here, the approach is however further simplified by considering a constant eddy viscosity, for which solutions are analytically tractable. Although this is a rather strong assumption, it is not unreasonable. For instance, using DNS data by Schlatter \& Örlü (2010) for $R e_{\theta}=4060$, we find that $0.06<\nu_{e} /\left(u_{\tau} \delta\right)<0.07$ for $0.2<z / \delta<0.6$, though $\nu_{e} /\left(u_{\tau} \delta\right)$ drops to 0.03 at $z / \delta=1$.

When $\nu_{t}=\nu+\nu_{e}$ is constant, $(2.14,2.15)$ simplify to

$$
\begin{aligned}
& \frac{\mathrm{d}^{2} \widetilde{\omega}_{3}}{\mathrm{~d} z^{2}}-\left(\imath U_{\infty} k_{1} / \nu_{t}+k^{2}\right) \widetilde{\omega}_{3}=0, \\
& \frac{\mathrm{d}^{4} \widetilde{u}_{3}}{\mathrm{~d} z^{4}}-\left(\imath k_{1} U_{\infty} / \nu_{t}+2 k^{2}\right) \frac{\mathrm{d}^{2} \widetilde{u}_{3}}{\mathrm{~d} z^{2}}+\left(\imath k_{1} U_{\infty} / \nu_{t}+k^{2}\right) k^{2} \widetilde{u}_{3}=0 .
\end{aligned}
$$

The first equation has two characteristic roots, i.e $\pm K= \pm k\left(1+\imath U k_{1} / k^{2} / \nu_{t}\right)^{1 / 2}$, or elaborated in its real and imaginary parts:

$$
K=k\left[\sqrt{\frac{1}{2}\left(1+\left(\frac{U_{\infty} k_{1}}{\nu_{t} k^{2}}\right)^{2}\right)^{1 / 2}+\frac{1}{2}}+\imath \sqrt{\frac{1}{2}\left(1+\left(\frac{U_{\infty} k_{1}}{\nu_{t} k^{2}}\right)^{2}\right)^{1 / 2}-\frac{1}{2}}\right]
$$

The second equation has four characteristic roots, i.e. $\pm k$ and $\pm K$. The root $K$ depends on $U_{\infty} k_{1} /\left(\nu_{t} k^{2}\right)$. Introducing $\ell_{k} \triangleq 2 \pi / k$, it can be elaborated as

$$
\frac{U_{\infty} k_{1}}{\nu_{t} k^{2}}=\frac{U_{\infty} \delta}{\nu_{t}} \frac{\ell_{k}}{2 \pi \delta} \frac{k_{1}}{k}=\left[\sqrt{\frac{c_{f}}{2}} \frac{\nu_{e}}{u_{\tau} \delta}+\frac{1}{R e}\right]^{-1} \frac{\ell_{k}}{2 \pi \delta} \frac{k_{1}}{k},
$$

with the Reynolds number $R e \triangleq U_{\infty} \delta / \nu$, and $c_{f}$ the skin friction coefficient. For $R e \rightarrow \infty$, it is expected that $\nu_{e} /\left(u_{\tau} \delta\right)=\mathcal{O}(1)$, so that $U_{\infty} k_{1} /\left(\nu_{t} k^{2}\right) \sim c_{f}^{-1 / 2}\left(\ell_{k} / \delta\right)\left(k_{1} / k\right)$. Other interesting limits correspond to $k_{1} / k \rightarrow 0$ and $\ell_{k} / \delta \rightarrow 0$. In both cases, $U_{\infty} k_{1} /\left(\nu_{t} k^{2}\right) \rightarrow 0$, and $K \rightarrow k$ (independent of Reynolds number).

Using the boundary conditions at $z=\infty$, and presuming $U_{\infty} k_{1} / k^{2} \neq 0$, solutions correspond to

$$
\widetilde{\omega}_{3}=A \exp (-K z), \text { and } \widetilde{u}_{3}=B \exp (-k z)+C \exp (-K z),
$$

with $A, B, C$ complex numbers that can only be determined if additional boundary conditions are known. For $U_{\infty} k_{1} / k^{2}=0, K=k$, and solutions correspond to

$$
\widetilde{\omega}_{3}=A \exp (-k z), \quad \text { and } \widetilde{u}_{3}=B z \exp (-k z)+C \exp (-k z)
$$

Moreover, for the limit of $k_{1} / k \rightarrow 0$ or $\ell_{k} / \delta \rightarrow 0,(2.20)$ converges to (2.21).

Finally, we note that for $\nu_{t}=0$, the potential-flow solution is recovered. Inserting $\nu_{t}=0$ in $(2.14,2.15)$ leads to $\tilde{\omega}_{3}=0$, while $\tilde{u}_{3}=A \exp (-k z)$. Interestingly, for the case of spanwise constant roughness $\left(k_{2}=0\right)$ and irrotational flow, Morgan \& McKeon (2018) mention an equivalent solution.

\section{Experimental verification}

In order to evaluate the relations derived above, experiments by Vanderwel \& Ganapathisubramani (2015) are examined. These experiments consist of a series of PIV 


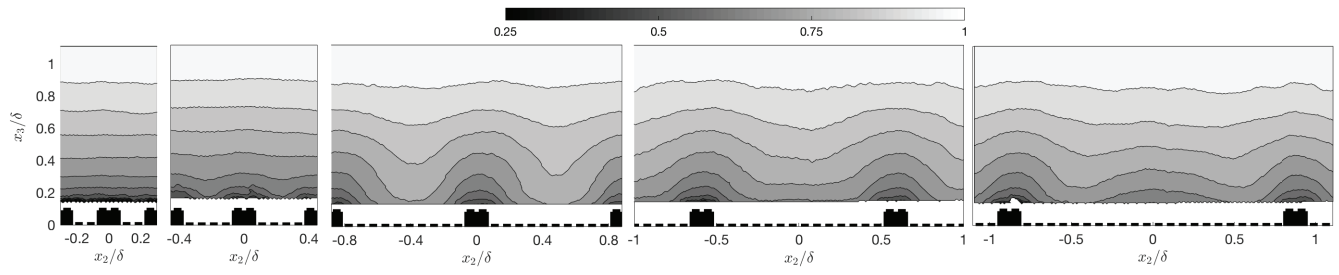

Figure 1: Overview of the experiments by Vanderwel \& Ganapathisubramani (2015). Left to right: contours of streamwise velocity $U / U_{\infty}$ shown in a spanwise-wall-normal plane obtained from the different experiments. Roughness elements are aligned in streamwise direction and have a different spanwise spacing per experiment. The shape of the elements is outlined at the bottom of the pictures.

measurements in rough-wall boundary layers with lego-brick roughness elements that are periodically organized with different spanwise spacings. An overview of the experiment and some mean velocity fields is shown in figure 1 . The roughness structure is such that $k_{1}=0$, while spanwise spacings of the different experiments correspond to $S / \delta=0.3$, $S / \delta=0.45, S / \delta=0.88, S / \delta=1.2, S / \delta=1.8$, with $S$ the wavelength and $\delta$ the boundary layer thickness. Velocity measurements are obtained in a wall-normal-spanwise plane, which allows to fully characterize the dispersive velocity field (given $k_{1}=0$ ). Full details of the experiment are found in Vanderwel \& Ganapathisubramani (2015).

The PIV measurement planes in the experiments extended approximately $240 \mathrm{~mm}$ in spanwise direction. The extent of these planes does not exactly correspond to an integer multiple of the spanwise roughness spacing (i.e, $S=32 \mathrm{~mm}, 48 \mathrm{~mm}, 96 \mathrm{~mm}$, $128 \mathrm{~mm}$, and $192 \mathrm{~mm}$ ) in the experiments. Therefore, the outer portions of the plane are truncated, and the velocity and Reynolds stress measurements are resampled in spanwise direction so that an integer number of spanwise periods and grid points is retained. Linear interpolation is used for the resampling; keeping the resolution as close as possible to the original. For the different cases $(S / \delta=0.3,0.45,0.88,1.2,1.8)$, this yields periods of 7 , $4,2,1$, and 1 , respectively. These results are then averaged in spanwise direction and used to obtain the dispersive stress fields as function of wall-normal direction.

First of all, in figure 2 an overview is provided of the total stresses as function of wall distance for the different experiments, as well as of the $\left\langle\bar{u}_{1}^{\prime \prime} \bar{u}_{1}^{\prime \prime}\right\rangle,\left\langle\bar{u}_{3}^{\prime \prime} \bar{u}_{3}^{\prime \prime}\right\rangle$, and $\left\langle\bar{u}_{1}^{\prime \prime} \bar{u}_{3}^{\prime \prime}\right\rangle$ dispersive stresses. Moreover, based on the maximum of the total stress, the skin friction coefficients for the various cases are also estimated. Figure 2 shows that the total stress depends non-monotonously on the spanwise roughness spacing, with the lowest skin friction at $S / \delta=0.3$, which increases to a maximum around $S / \delta=0.88$, and subsequently decreases again when $S / \delta$ is further increased to 1.8. Similar differences in skin friction as function of spanwise spacing were, e.g., also observed by Medjnoun et al. (2018); Hwang \& Lee (2018).

Further in figure $2(\mathrm{c}-\mathrm{d})$, it is observed that the maximum magnitude of the dispersive stresses (observed around $z / \delta \approx 0.2$ ) are reasonably correlated with the total skin friction, in particular for the cases with $S / \delta \leqslant 0.88$. For wider spacings, trends are less clear. Differences in dispersive stresses result from processes induced by the shape and spacing of the roughness elements as well as the flow in the inner layer of the boundary layer. They essentially serve as a boundary condition for the outer layer decay relations derived in $\S 2$.

Finally, it is seen in figure $2(\mathrm{c}-\mathrm{d})$ that the dispersive stresses roughly decay exponentially with increasing wall-normal distance as suggested by the analysis in $\S 2$. However, 


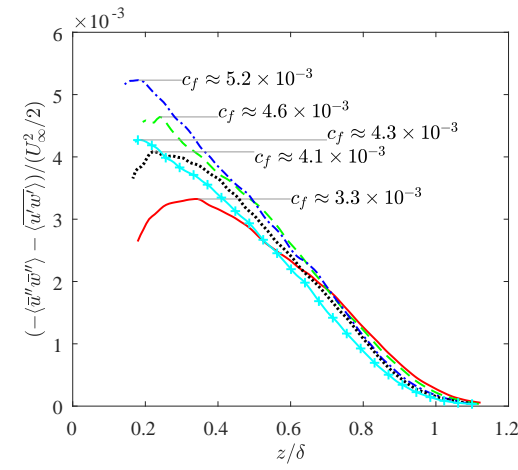

(a)
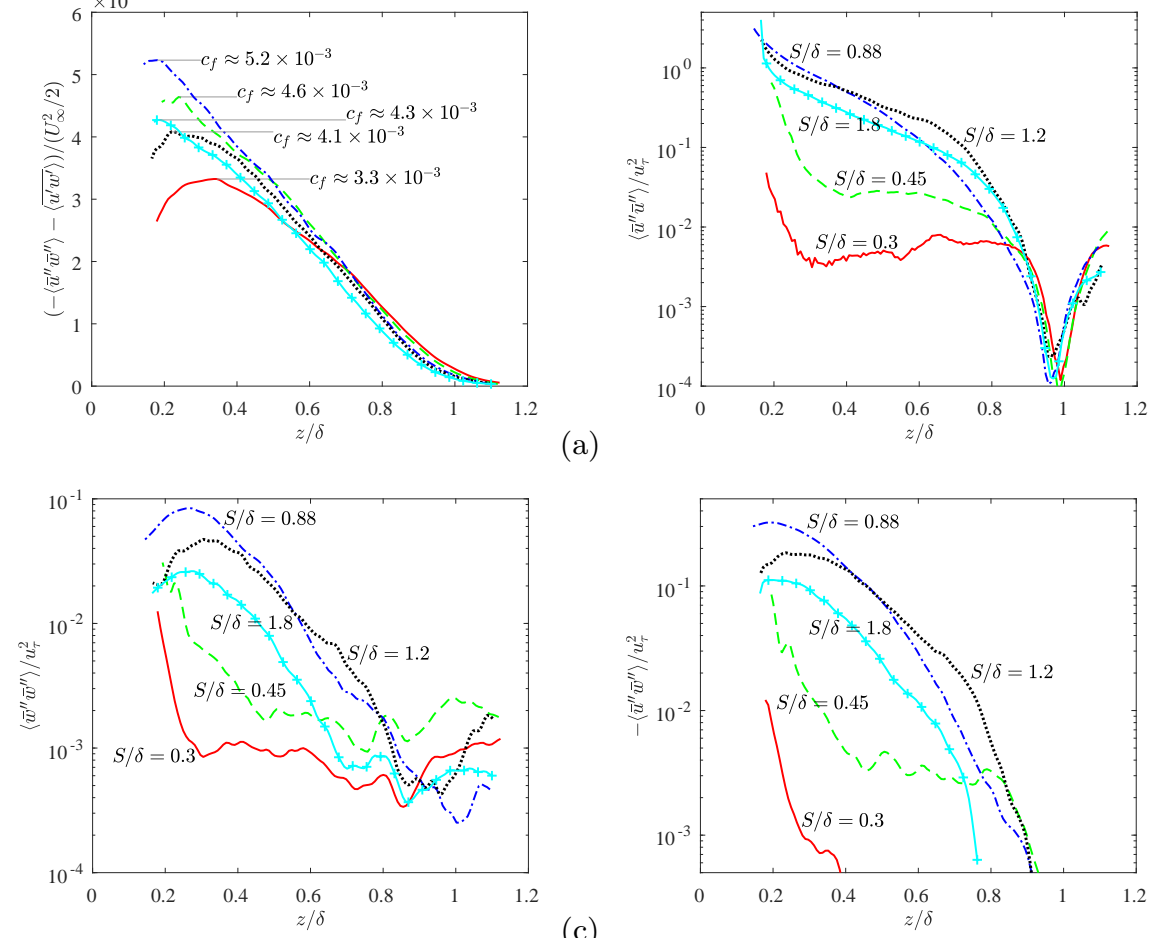

(b)

(c)

Figure 2: (a) Total stress as function of wall distance, normalized by dynamic pressure. (b,c,d) $\left\langle\bar{u}_{1}^{\prime \prime} \bar{u}_{1}^{\prime \prime}\right\rangle,\left\langle\bar{u}_{3}^{\prime \prime} \bar{u}_{3}^{\prime \prime}\right\rangle$, and $-\left\langle\bar{u}_{1}^{\prime \prime} \bar{u}_{3}^{\prime \prime}\right\rangle$ respectivelly, as function of wall distance. Linetypes $(-,--,-, \cdots,-+)$ correspond to different spacings.

there is no clear single slope to be identified, as the stresses result from a sum over all the modes present in the flow; each decaying at their own rate. To this end, the decay should be analyzed mode by mode (cf. below). It is further acknowledged that the decay saturates towards the top of the boundary layer, as measurement noise starts to play an important role in the very small remaining dispersive stress components. Exploring this error in detail is beyond the scope of the current study. However, we obtain a direct estimate of the error by looking at the free-stream level (at $z / \delta \approx 1.1$ ) of the dispersive stresses $\left\langle\bar{u}^{\prime \prime} \bar{u}^{\prime \prime}\right\rangle$ and $\left\langle\bar{w}^{\prime \prime} \bar{w}^{\prime \prime}\right\rangle$, corresponding respectively to $5 \times 10^{-3} u_{\tau}^{2}$ and $10^{-3} u_{\tau}^{2}$. At this location dispersive stresses are expected to be zero, so that the observed magnitudes correspond to the respective error levels. It is seen that the error on $\left\langle\bar{u}^{\prime \prime} \bar{u}^{\prime \prime}\right\rangle$ is larger than that on $\left\langle\bar{w}^{\prime \prime} \bar{w}^{\prime \prime}\right\rangle$. This is consistent with the measurement set-up where $w$ is an in-plane component that can be estimated directly while $u$ is the out-ofplane component that has to be reconstructed by combining the displacements observed by the two cameras in the PIV set-up (Vanderwel \& Ganapathisubramani 2015). The ratio of the uncertainty in the out-of-plane component to the uncertainty of the in-plane components is approximately $1 / \tan \theta$, where $\theta$ is the included half angle of the cameras (see Prasad 2000 for details). In the set-up of Vanderwel \& Ganapathisubramani (2015), $\theta$ is 35 degrees, so that the uncertainty ratio between $u$ and $w$ components is 1.43 , or approximately 2 when comparing $\left\langle\bar{u}^{\prime \prime} \bar{u}^{\prime \prime}\right\rangle$ and $\left\langle\bar{w}^{\prime \prime} \bar{w}^{\prime \prime}\right\rangle$.

Now turning to the evaluation of the decay of dispersive stresses per mode, a Fourier transform in spanwise direction on the dispersive velocity field $\overline{\boldsymbol{u}}^{\prime \prime}$ is performed. The 
decay of the spectra of stream and wall-normal dispersive velocity components is the focal point, respectively defined as $S_{u u}\left(k_{2}, z\right)=\bar{u}_{1}\left(k_{2}, z\right) \bar{u}_{1}^{*}\left(k_{2}, z\right)$ and $S_{w w}\left(k_{2}, z\right)=$ $\bar{u}_{3}\left(k_{2}, z\right) \bar{u}_{3}^{*}\left(k_{2}, z\right)$, where $*$ is used for the complex conjugate. Given the relations $(2.21)$ and (2.10),

$$
\begin{array}{r}
S_{u u}=D_{1} \exp \left(-2 k_{2} z\right), \\
S_{w w}=\exp \left(-2 k_{2} z\right)\left(D_{2}+D_{3} z+D_{4} z^{2}\right),
\end{array}
$$

is expected, where $D_{1}-D_{4}$ are constants that can, e.g., be found by matching the linear solution to the near wall nonlinear solution at a location sufficiently far from the wall.

In figure 3, decay of $S_{u u}$ (left panels) and $S_{w w}$ (right panels) is shown as function of wall distance for the different cases (parts a-e). Results are shown in semi-log scale, and up to four modes are shown, corresponding to those modes that most contribute to the $\left\langle\bar{u}_{1}^{\prime \prime} \bar{u}_{3}^{\prime \prime}\right\rangle$ dispersive stress at $z=0.2 \delta$. Next to that, in the left panels the slopes $-2 k_{2}$ (corresponding to (3.1)) are also plotted for the different modes, while in the right panel least-squares fits of $(3.2)$ over the range $0.2<z / \delta<1.0$ are shown. Note that in figure $3(\mathrm{a}-\mathrm{c})$ only two and three modes are shown, as other contributing modes fall within the noise level of the measurements. Similar to before, a dynamic range of two to three decades in the spectra is observed, but for modes with less energy content, the dynamic range can be significantly lower (see, e.g., mode $k \delta /(2 \pi)=5.1$ in figure $3(\mathrm{e})$ ). In order to visually illustrate the noise level of the measurements relative to the magnitude of the different modes, a marker on each mode indicating the level $10^{-3} u_{\tau}^{2}$ relative to the scaling used for the modes is included. Note that this represents the estimated error level for $S_{w w}$, while the error on $S_{u u}$ is two to five times higher (cf. discussion above).

Looking at the right panels of figure 3 , it is observed that the fits of $S_{w w}$ match the data very well between $z / \delta=0.2$ and $z / \delta=0.6$ for all cases and modes. At higher wall distances, the measurements are saturated with noise, such that no meaningful comparison can be performed. Considering the matching of $\exp \left(-2 k_{2} z\right)$ to $S_{u u}$ in the left panels of figure 3, the picture is more diverse. For the first two cases (panel a and b), a good agreement is found between $z / \delta=0.2$ and $z / \delta=0.3$ to 0.4 . Beyond that, the noise level starts to dominate the measurements. For the other cases (panel c- d), an adequate matching for higher modes is generally found, i.e. in particular for $k \delta /(2 \pi)=\delta / \ell_{k}>2$. For lower modes, the experimental slopes do not correspond well with the analytical slope, and are typically lower in absolute value.

\section{Discussion}

Given the strong assumptions made in the development of analytical solutions in $\S 2.4$, the correspondence with experiments is good, in particular when considering $S_{w w}$. The results for $S_{u u}$ are mixed, and differences could be attributed to nonlinear effects, the lack of streamwise mean-flow homogeneity, or using $\Gamma=0$ in the linearisation, as further discussed below.

First of all, when considering the quality of fits in section 3, nonlinear effects can play a role, in particular since modes that are poorly predicted tend to occur mainly for $S / \delta=0.88,1.2$, and 1.8 , which have the strongest dispersive shear stresses relative to the friction velocity (see figure 2). Values for these spacings correspond respectively to $30 \%, 18 \%$, and $11 \%$, versus $9 \%$, and $1 \%$ for $S / \delta=0.45$, and 0.3 . Nevertheless, since also the former values remain relatively small, and since $S_{w w}$ fits the data reasonably well, other effects (discussed below) are seemingly more probable. Furthermore, contributions 

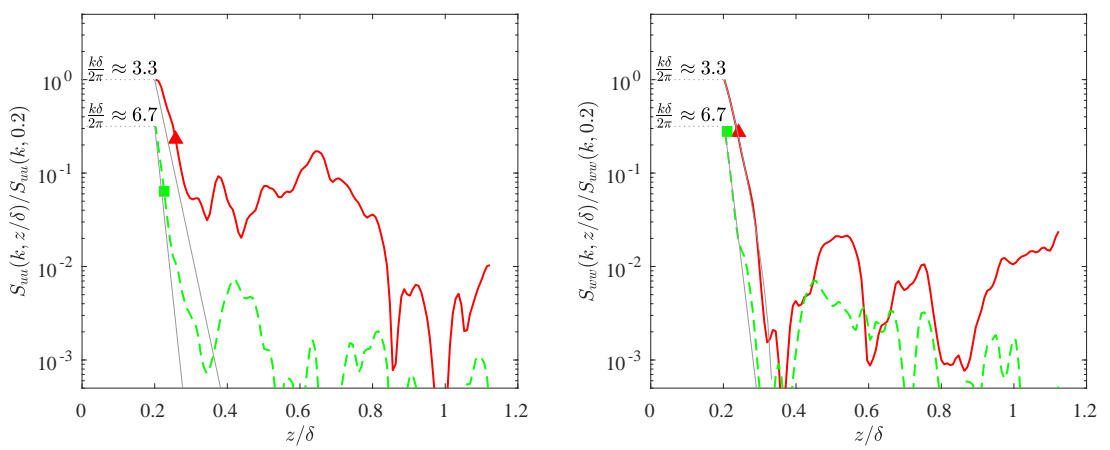

(a)
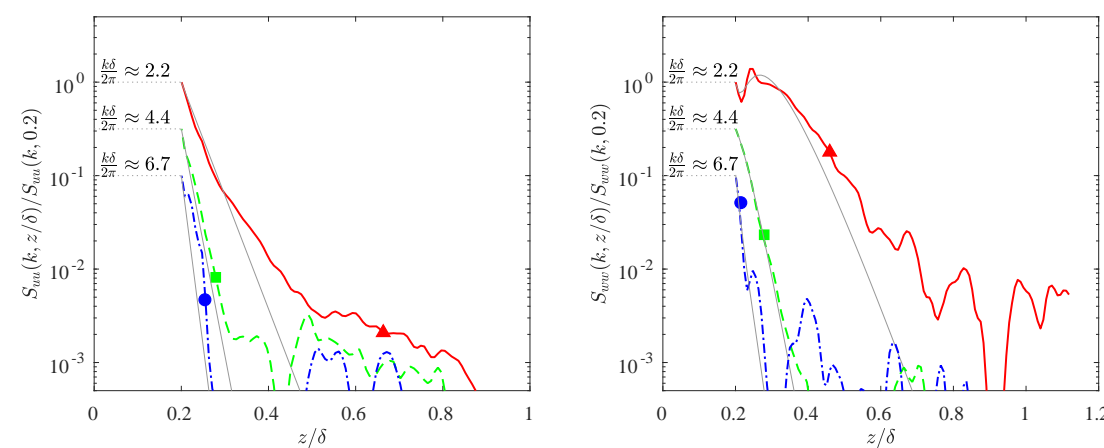

(b)
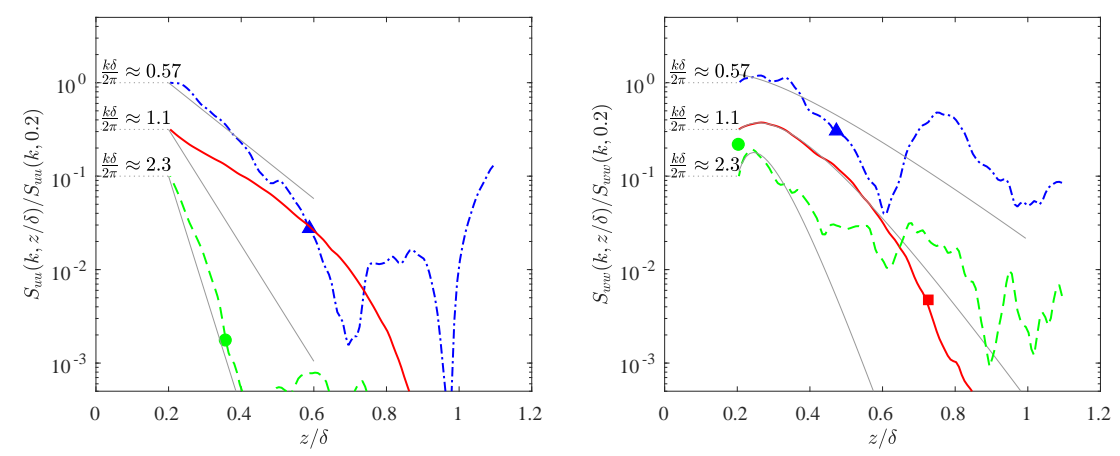

(c)

Figure 3: (continued on next page) Decay of Fourier modes of streamwise (left) and normal (right) dispersive components as function of wall distance $z / \delta$. (a) $S / \delta=0.3$ (b) $S / \delta=0.45 ;$ (c) $S / \delta=0.88$; (d) $S / \delta=1.2 ;$ (e) $S / \delta=1.8$. $(-\boldsymbol{\Delta},--\mathbf{\square},-\cdot \bullet, \cdots \bullet)$ : up to four Fourier modes with highest contribution to the dispersive shear component $\left\langle f u_{1}^{\prime \prime} f u_{3}^{\prime \prime}\right\rangle$; line types are ordered according to magnitude of the contributing mode. Lines are shifted with half a decade for visibility. (gray line in left plots): slope corresponding to $\exp \left(-2 k_{2} z\right)$. (gray line in right plots): least-squares fit of $\exp \left(-2 k_{2} z\right)\left(D_{2}+D_{3} z+D_{4} z^{2}\right.$ ) to the data in the range $0.2<z<1.0 .(\boldsymbol{\Delta}, \mathbf{\square}, \bigcirc, \boldsymbol{\vee})$ : markers on the different Fourier modes are inserted at level $10^{-3} u_{\tau}^{2} / S_{u u}(k, 0.2)$ and $10^{-3} u_{\tau}^{2} / S_{w w}(k, 0.2)$ respectively.

from $\bar{u}^{\prime \prime} \bar{u}^{\prime \prime}$, which are much larger relative to the friction velocity (see figure $2 \mathrm{~b}$ ), do not play a role in the nonlinear equations $(2.3)$ when $k_{1}=0$.

A further assumption in the derivation of $(2.14,2.15)$ is that the streamwise evolution of mean velocity components can be neglected in (2.1) and (2.3). For $k_{1} \rightarrow 0$, this 

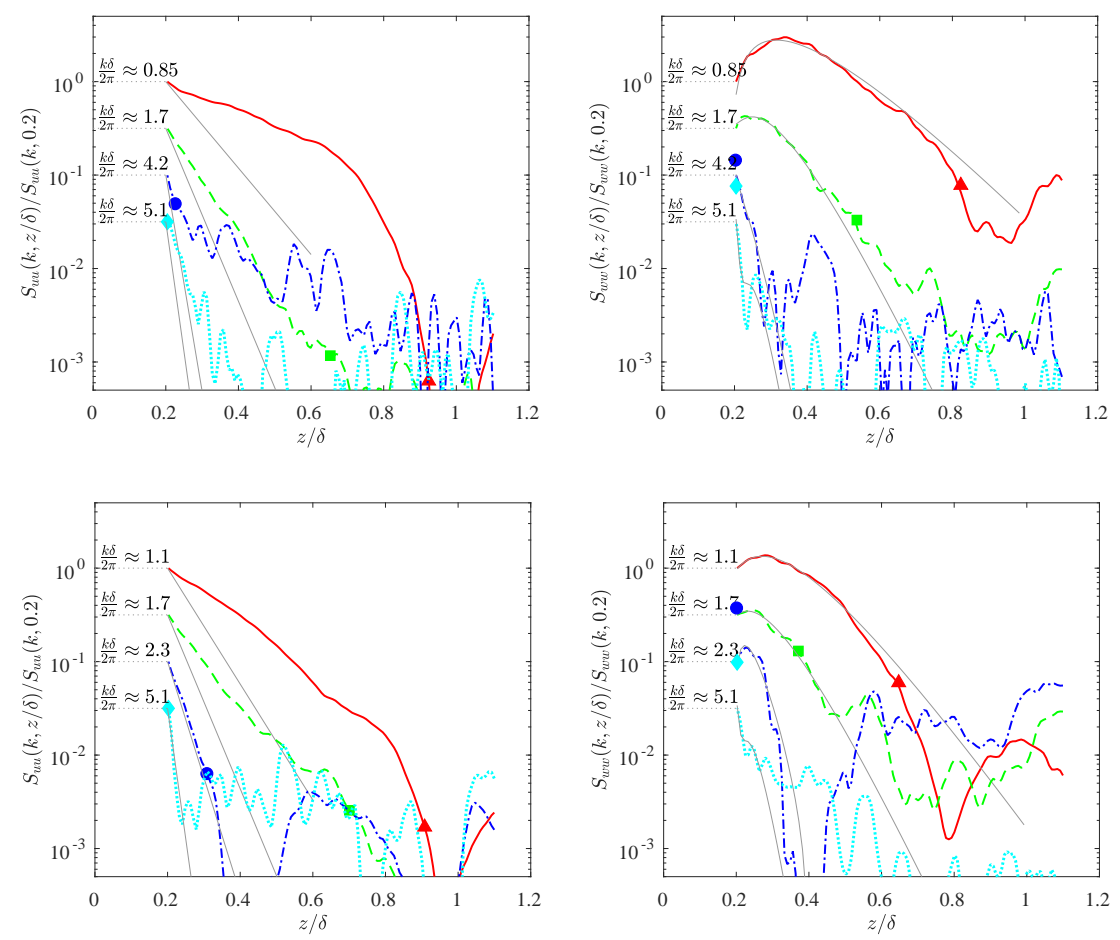

(e)

Figure 3: (continued from previous page)

assumption does not hold. Consequently, the limit $k_{1} / k \rightarrow 0$ in (2.18) is not viable, and only $\ell_{k} / \delta \rightarrow 0$ (and thus, $\left.k \delta \rightarrow \infty\right)$ may be expected to lead to (2.21). The picture in figure 3 above is consistent with that, showing a good match between theory and results for $\delta / \ell_{k}>2$. This is also in agreement with results from Vanderwel \& Ganapathisubramani (2015) and Hwang \& Lee (2018) that show that the excitation of secondary motions is most effective around a spanwise roughness spacing of $S / \delta=\mathcal{O}(1)$, but decreases drastically when $S$ is decreased.

Thirdly, also $\Gamma \approx 0$ and $U \approx U_{\infty}$ are strong simplifications in the derivation of $(2.14,2.15)$ : e.g., around $z / \delta=0.2$, the velocity deficit is already about $30 \%$ of the free-stream velocity. Interestingly, for $k_{1} / k \rightarrow 0$, the effect of $\Gamma$ in (2.13) disappears, so that (2.15) and solutions for $\tilde{u}_{3}$ remain the same when $\Gamma \neq 0$. This is not the case for (2.13), i.e. $\Gamma \neq 0$ leads to an additional term $\imath k_{2} \tilde{u}_{3} \Gamma$ in (2.14). Thus related solutions for $\omega_{3}$ and $u_{1}$ may depend on $u_{3}$ and $\Gamma$. For $\ell_{k} / \delta \rightarrow 0(k \rightarrow \infty)$, the relative importance of the additional term $\imath k_{2} \tilde{u}_{3} \Gamma$ decreases in (2.14), since other terms scale with $k^{2}$. This remains consistent with observations in section 3 , that $S_{u u}$ matches data better when $\ell_{k} / \delta$ is sufficiently small. It should be noted that for $\Gamma \neq 0$, the dispersive eddy viscosity $\nu_{e}^{\prime \prime}$ and the last term on the right-hand side of (2.7) may also become important; finding exact (linear) expressions for this term is however akin to the classical turbulence closure problem, and not further considered here.

The current analytical model may shed some further light on the characterization of roughness length scales in rough boundary layers. Whereas the classical roughness 'height' is directly associated with the skin friction drag, the horizontal roughness length $\ell_{k}$ dictates how fast dispersive flow perturbations decay in the outer layer, supporting 
the notion of spanwise homogeneous outer flow behavior that is independent of the nearwall flow when $\ell_{k}$ is sufficiently small. Current findings may also be of interest for the development of rough-wall stress conditions for simulations, in particular in situations with multi-scale roughness (e.g. prevalent in the atmospheric boundary layer) in which only part of the roughness is resolved by the mesh (Anderson \& Meneveau 2011).

\section{Summary}

A framework is presented to study the decay of dispersive stresses in the outer layer of a rough-wall boundary layer by using a linearization of the Navier-Stokes equations. By formulating the problem in Fourier space, this leads to two coupled ordinary differential equations per horizontal wavevector. These equations require the unperturbed background velocity $U(z)$ and eddy viscosity $\nu(t)$ as input, and are expected to be exact to first order when $\Gamma \ll 1$, as is the case near the top of the boundary layer. A further approximation that uses $\Gamma=0$, and assumes a constant eddy viscosity allows for solutions that are analytically tractable. These solutions were compared to the experimental data from Vanderwel \& Ganapathisubramani (2015), showing a reasonable agreement with the measurements given the assumptions that were made to obtain the analytical model.

In the future, it will be of interest to investigate more involved solutions of $(2.14,2.15)$ using detailed parametrizations of $\nu_{e}(z)$ in the outer layer of a boundary layer, or by directly solving the coupled system $(2.12,2.13)$ using an additional parametrization of $\Gamma(z)$ and $U(z)$. In this case, analytically tractable solutions may not anymore exist, but numerical solutions should be obtained without much complication. In this context, more extensive comparison with data, in particular including also roughness elements with $k_{1} \neq 0$ is also relevant.

JM acknowledges support from the Research Foundation - Flanders (FWO Grant no. V4.255.17N). BG acknowledges the financial support from Engineering and Physical Sciences Research Council of the United Kingdom (EPSRC grant ref. no. EP/R034370/1 and EP/P021476/1).

\section{REFERENCES}

Anderson, W., Barros, J. M., Christensen, K. T. \& Awasthi, A. 2015 Numerical and experimental study of mechanisms responsible for turbulent secondary flows in boundary layer flows over spanwise heterogeneous roughness. J. Fluid Mech. 768, 316-347.

Anderson, W. \& Meneveau, C. 2011 Dynamic roughness model for large-eddy simulation of turbulent flow over multiscale, fractal-like rough surfaces. J. Fluid Mech. 679, 288-314.

Barros, J. M. \& Christensen, K. T. 2014 Observations of turbulent secondary flows in a rough-wall boundary layer. J. Fluid Mech. 748, R1.

CAstro, I. P. 2007 Rough-wall boundary layers: mean flow universality. J. Fluid Mech. 585, 469-485.

Chan, L., MacDonald, M., Chung, D., Hutchins, N. \& Ooi, A. 2018 Secondary motion in turbulent pipe flow with three-dimensional roughness. J. Fluid Mech. 854, 5-33.

Deser, S. 1967 Covariant decomposition of symmetric tensors and the gravitational Cauchy problem. Annales de l'Inst. Henri Poincaré, section A 7, 149-188.

Finnigan, J. 2000 Turbulence in plant canopies. Annu. Rev. Fluid Mech. 32 (1), 519-571.

Flack, K. A., Schultz, M. P. \& Connelly, J. S. 2007 Examination of a critical roughness height for outer layer similarity. Phys. Fluids 19 (9), 095104.

HwANG, H. G. \& LEE, J. H. 2018 Secondary flows in turbulent boundary layers over longitudinal surface roughness. Phys. Rev. Fluids 3, 014608.

JimÉnEZ, J. 2004 Turbulent flows over rough walls. Annu. Rev. Fluid Mech. 36, 173-196. 
JimÉnez, J. 2016 Optimal fluxes and Reynolds stresses. J. Fluid Mech. 809, 585-60.

Kevin, K., Monty, J. P., Bai, H. L., Pathikonda, G., Nugroho, B., Barros, J. M., Christensen, K. T. \& Hutchins, N. 2017 Cross-stream stereoscopic particle image velocimetry of a modified turbulent boundary layer over directional surfacepattern. $J$. Fluid Mech. 813, 412-435.

Manes, C., Pokrajac, D., Coceal, O. \& McEwan, I. 2008 On the significance of forminduced stress in rough wall turbulent boundary layers. Acta Geophysica 56 (3), 845-861.

Medjnoun, T., Vanderwel, C. \& Ganapathisubramani, B. 2018 Characteristics of turbulent boundary layers over smooth surfaces with spanwise heterogeneities. J. Fluid Mech. 838, 516-543.

Morgan, J. \& McKeon, B. J. 2018 Relation between a singly-periodic roughness geometry and spatio-temporal turbulence characteristics. Int. J. Heat Fluid Fl. 71, $322-333$.

Nezu, I. \& Nakagawa, H. 1984 Cellular secondary currents in straight conduit. J. Hydraul. Eng. 110 (2), 173-193.

Nikora, V., Goring, D., McEwan, I. \& Griffiths, G. 2001 Spatially averaged open-channel flow over rough bed. Journal of Hydraulic Engineering 127 (2), 123-133.

Nikora, V., McEwan, I., Mclean, S., Coleman, S., Pokrajac, D. \& Walters, R. 2007 Double-averaging concept for rough-bed open-channel and overland flows: Theoretical background. Journal of Hydraulic Engineering 133 (8), 873-883.

Prasad, A. K. 2000 Stereoscopic particle image velocimetry. Experiments in Fluids 29 (2), $103-116$.

Raupach, M. R., Antonia, R. A. \& Rajagopalan, S. 1991 Rough-wall turbulent boundary layers. Applied Mechanics Reviews 44 (1), 1-25.

Raupach, M. R. \& Shaw, R. H. 1982 Averaging procedures for flow within vegetation canopies. Boundary-Layer Meteorology 22 (1), 79-90.

Schlatter, P. \& ÖRLü, R. 2010 Assessment of direct numerical simulation data of turbulent boundary layers. Journal of Fluid Mechanics 659, 116-126.

Townsend, A. A. 1956 The Structure of Turbulent Shear Flow, 1st edn. Cambridge University Press.

Townsend, A. A. 1976 The Structure of Turbulent Shear Flow, 2nd edn. Cambridge University Press.

Vanderwel, C. \& Ganapathisubramani, B. 2015 Effects of spanwise spacing on large-scale secondary flows in rough-wall turbulent boundary layers. J. Fluid Mech. 774 (R2), 1-11.

WANG, Z.-Q. \& Cheng, N.-S. 2005 Secondary flows over artificial bed strips. Advances in Water Resources 28 (5), 441-450.

Wu, J.-Z., ZноU, Y. \& Wu, J.-M. 1996 Reduced stress tensor and dissipation and the transport of lamb vector. Tech. Rep. 96-21. ICASE.

YANG, J. \& ANDERSON, W. 2018 Numerical study of turbulent channel flow over surfaces with variable spanwise heterogeneities: Topographically-driven secondary flows affect outerlayer similarity of turbulent length scales. Flow Turbul. Combust. 100 (1), 1-17. 